\title{
A Novel Detection and Classification Algorithm for Power Quality Disturbances using Wavelets
}

\author{
${ }^{1}$ C. Sharmeela, ${ }^{2}$ M.R. Mohan, ${ }^{2}$ G.Uma and ${ }^{2}$ J.Baskaran \\ ${ }^{1}$ A.C. College of Technology, Anna University \\ ${ }^{2}$ DEEE, College of Engineering, Anna University, Chennai-600 025, India
}

\begin{abstract}
This study presents a novel method to detect and classify power quality disturbances using wavelets. The proposed algorithm uses different wavelets each for a particular class of disturbance. The method uses wavelet filter banks in an effective way and does multiple filtering to detect the disturbances. A qualitative comparison of results shows the advantages and drawbacks of each wavelet when applied to the detection of the disturbances. This method is tested for a large class of test conditions simulated in MATLAB. Power quality monitoring together with the ability of the proposed algorithm to classify the disturbances will be a powerful tool for the power system engineers.
\end{abstract}

Key words: Wavelet transforms, power quality disturbances, transients, voltage sag, voltage swell

\section{INTRODUCTION}

Electric power quality is an important issue in power systems nowadays .The demand for clean power has been increasing in the past several years. The reason is mainly due to the increased use of microelectronic processors in various types of equipments such as computer terminals, programmable logic controllers and diagnostic systems. Most of these systems are quite susceptible to disturbances in the supply voltage. For example a momentary power interruption or thirty percent voltage sag lasting for hundredth of a second can reset the PLCs in an assembly line. The amount of waveform distortion has been found to be more significant nowadays due to the wide applications of nonlinear electronic devices in power apparatus and systems.

Without determining the existing levels of power quality, electric utilities cannot adopt suitable strategies to provide a better service. Therefore an efficient approach of justifying these electric power quality disturbances is motivated. Several research studies regarding the power quality have been conducted. Their aims were often concentrated on the collection of raw data for a further analysis, so that the impacts of various disturbances can be investigated. Sources of such disturbances can be located or further mitigated. However, the amount of acquisition data was often massive in their test cases. Such an abundance of data may be time consuming for the inspection of possible culprits. A more efficient approach is thus required in the power quality assessment.

The implementation of the discrete Fourier transform by various algorithms has been constructed as the basis of modern spectral analysis. Such transforms were successfully applied to stationary signals where the properties of signals did not evolve in time. However, for those non-stationary signals any abrupt change may spread over the whole frequency axis. In this situation, the Fourier transform is less efficient in tracking the signal dynamics ${ }^{[1]}$. A point -topoint comparison scheme has been proposed to discover the dissimilarities between consecutive cycles $^{[2]}$. This approach was feasible in detecting certain kinds of disturbances but fail to detect those disturbances that appear periodically. With the introduction of new network topologies and improved training algorithms, neural network technologies have demonstrated their effectiveness in several power system applications ${ }^{[3]}$. Once the networks have been well trained, the disturbances that correspond to the new scenario can be identified in a very short time ${ }^{[4]}$.

This technique has also been applied in the power system applications. However, it can only be applied to detect a particular type of disturbance. When encountering different disturbances, the network structure has to be reorganized, plus the training process must be restarted. A method of detecting power quality disturbances based on neural networks and wavelets has been proposed ${ }^{[7]}$. In this method, the fundamental component is removed using wavelets and the remaining signal corresponding to disturbances is processed and given as input to ANN. However, this method fails to detect voltage sag/swell and also new ANN's have to be developed for different rated load voltages and sampling frequencies.

Recently with the emergence of wavelets it has paved a unified framework for signal processing and its applications ${ }^{[5]}$. Fourier transforms rely on a uniform window for spreaded frequencies. Wavelet transforms can apply various lengths of windows according to the amount of signal frequencies. Characteristics of non-

Corresponding Author: $\quad$ C. Sharmeela, A.C. College of Technology, Anna University, DEEE, College of Engineering, Anna University, Chennai-600 025, India 
stationary disturbances were found to be more closely monitored by wavelets. The transient behavior, cavities and discontinuities of signals can be all investigated by wavelet transforms. For example, if there is an instantaneous impulse disturbance, which happens at a certain time interval it may contribute to the Fourier transform, but its location on the time axis is lost. However, by wavelets both time and frequency information can be obtained. In other words, the wavelet transform are more local. Instead of transforming a pure 'time domain' in to a pure' frequency domain ', the wavelet transforms find a good compromise in time - frequency domain.

This paper presents novel algorithm, which overcomes all these difficulties and can accurately detect and classify the disturbances present in the signal. This method is independent of the load voltage and can be easily customized for different sampling frequencies. In this approach, for detecting each disturbance a particular wavelet is used. The method uses wavelet filter banks in an effective way and does multiple filtering to detect the disturbances. The performance evaluation of different wavelets in the proposed method shows the capability of a particular wavelet in detecting a particular disturbance.

\section{Algorithm for detection and classification}

Event detection and classification: In this scheme multiple filtering is applied to the signal and the resulting filtered signals are processed to detect and classify the disturbances present in the signal. In the proposed method four filtered signals are obtained using wavelet filter banks by applying four different levels of decomposition and reconstruction to the signal. The disturbances are classified according to frequencies that characterize the power quality events. By applying appropriate levels of decomposition and reconstruction to the signal, the resulting reconstructed signal can used to analyze the presence of a particular class of disturbance. Thus different levels of decomposition and reconstruction are applied to the signal for different classes of disturbances.

A level 1 details of the input signal is found using Db3 wavelet and this filtered signal is analyzed tocheck the presence of transient disturbances. The level 5 details of the input signal is found using Dmey wavelet and this filtered signal is analyzed to check the presence of harmonics. The level 8 approximations of the input signal is obtained using $\mathrm{Db} 10$ or sym 8 and this filtered signal is analyzed to check the presence of voltage flicker. Level 5 approximations of the input signal is found. The approximated signal is free from any harmonic components and this filtered signal is analyzed to check the presence of voltage sag/swell.

The first three filtered, reconstructed signals are converted to pulses of unique amplitude by comparing with $\mathrm{Thresh}_{\mathrm{p}}$ limits and thereby noises are removed. This Thresh $\mathrm{p}$ limit is given by $\alpha *$ load voltage.

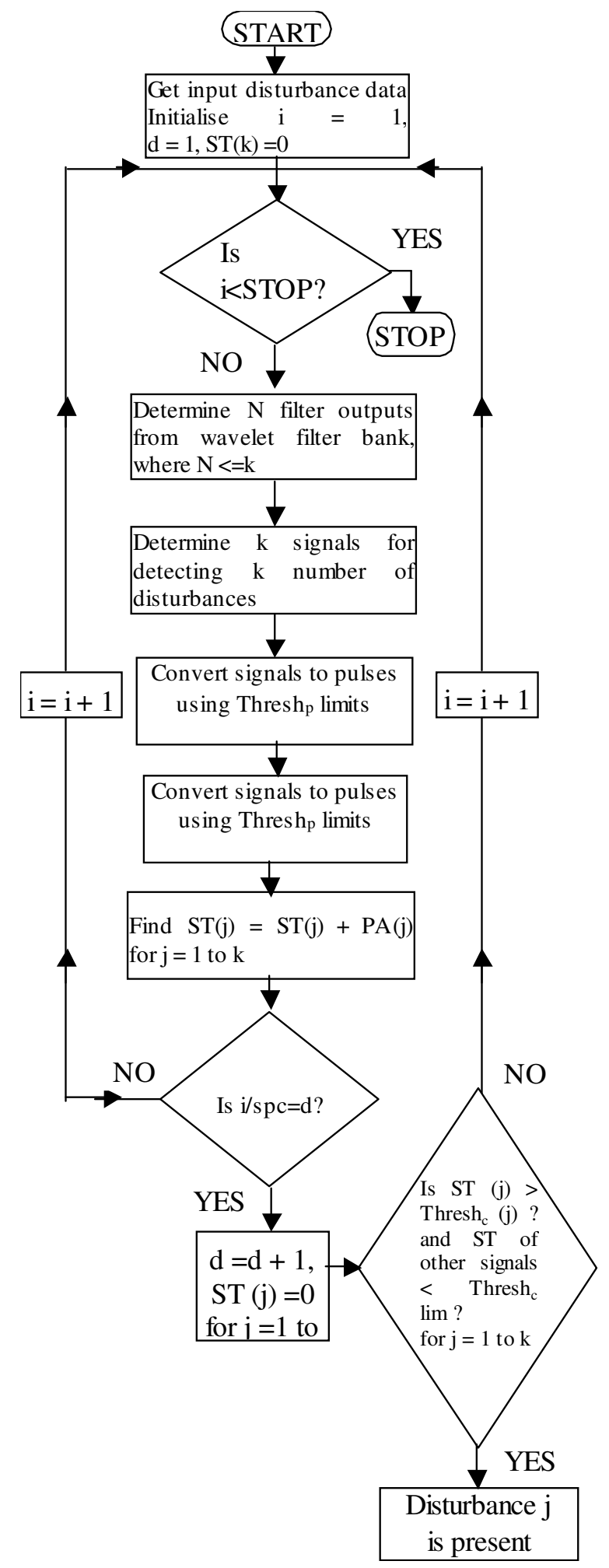

Fig. 1: Flowchart for the proposed detection algorithm

The value of $\alpha$ is found out by trial and error by testing for different cases of disturbance data. For the fourth filtered reconstructed signal the level 5-approximation signal is compared with the reference signal and two 
signals are obtained. One signal contains pulses corresponding to the presence of voltage swell and the other contains pulses corresponding to the presence of voltage sag. Five signals are obtained, one each for a disturbance from the four filter outputs. The sum total of pulses in each signal is calculated for one cycle.

A threshold limit is fixed for the sum total in one cycle for each disturbance. This is named as $\mathrm{Thresh}_{\mathrm{c}}$ limits. These $\mathrm{Thresh}_{\mathrm{c}}$ limits are given by $\beta^{*}$ sampling frequency. The value of $\beta$ is found out by trial and error by testing for different disturbance cases. A disturbance is said to occur if the sum total in the signal corresponding to the same disturbance after every one cycle is greater than the threshold limit kept for it and simultaneously the sum total of the pulses in the other signals for the same cycle are less than their threshold limits. Thus the type and time of occurrence of the disturbance can be found. The detection algorithm is explained using the flowchart given in Fig. 1

In Fig. 1:

$\mathrm{Spc}=$ Samples per cycle

ST=Sum total

$\mathrm{K}=$ number of disturbances considered.

PA=Pulse Amplitude.

Stop=Last sample indicating the end of the signal

Choice of mother wavelet: In the fast transient case, the waveforms are marked with sharp edges, abrupt and rapid changes and a fairly short duration in time. In this case $\mathrm{Db} 3$ and $\mathrm{Db} 4$ are particularly good in detecting these disturbances. In slow transient case, the waveforms are marked with a slow change or smooth amplitude change. $\mathrm{Db} 3$ and $\mathrm{Db} 4$ cannot catch those disturbances because the time interval integral is very short.

However if $\mathrm{Db} 8, \mathrm{Db} 10$ and sym 8 are used the time interval integral is long enough and thus such wavelets can sense the slow changes. Thus in detecting sags which are not sudden Db10, sym8 and Db8 can be used. For detecting harmonics Dmey gives best results and for transients $\mathrm{Db} 3$ can be used.

Disturbances considered for analysis: Five different disturbance categories are examined here. These are some interesting and most common cases. A brief explanation of the five categories addressed in this study is given.

Voltage sag is described as a drop of $10-90 \%$ of the rated system voltage lasting for half a cycle to $1 \mathrm{~min}$. The causes of voltage sags are caused by system faults and energisation of heavy loads. A $40 \%$ voltage Sag lasting for 2 cycles is shown in the Fig. 2a.

Voltage swells are defined as the increase of fundamental frequency voltage for a short duration lasting for half a cycle to $1 \mathrm{~min}$. The typical values are $110-180 \%$ of the rated system voltage.A $40 \%$ swell disturbance lasting for $40 \mathrm{~ms}$ is simulated and shown in Fig. 2b.

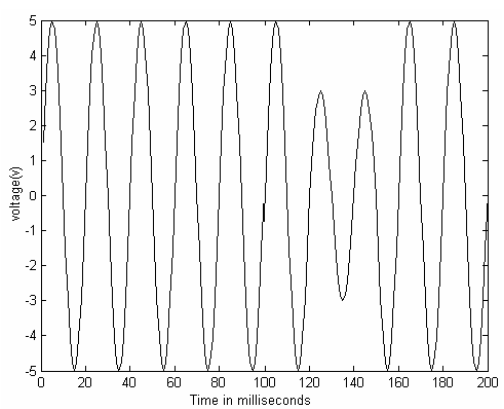

(a) time (ms)

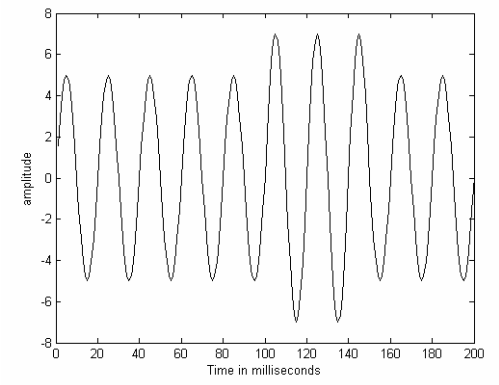

(b) time (ms)

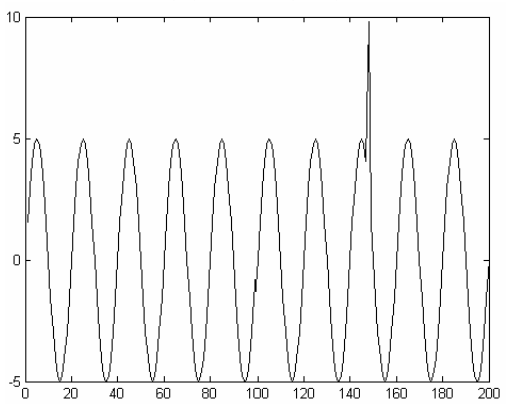

(c) time (ms)

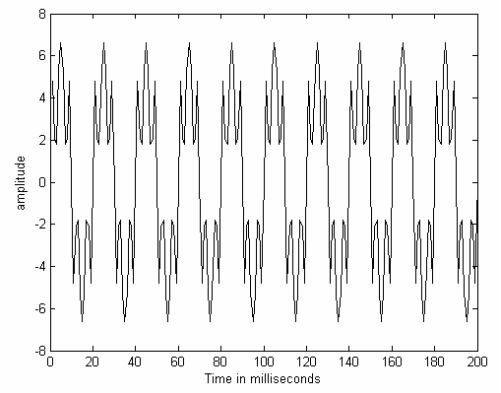

(d) time (ms)

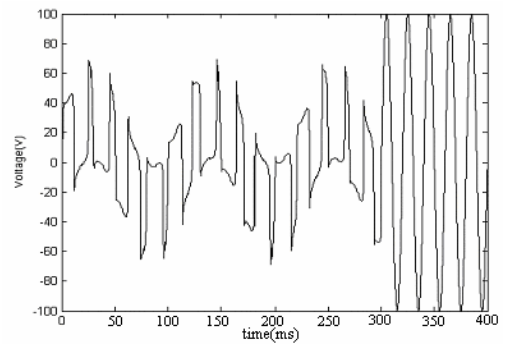

(e) time (ms)

Fig. 2: The various power quality disturbances, (a) Voltage Sag (b) Voltage Swell (c) Transient, (d) Harmonics (e) Voltage flicker 
Am. J. Appl. Sci., 3 (10): 2049-2053, 2006

\begin{tabular}{|c|c|c|c|c|c|}
\hline Wavelet & Impulsive Transient & Harmonics & Flicker & Voltage Sag & Voltage Swell \\
\hline Dmey & $\begin{array}{l}\text { Detects but not so } \\
\text { accurate compared } \\
\text { to Db3 }\end{array}$ & $\begin{array}{l}\text { Detects and classifies } \\
\text { accurately }\end{array}$ & $\begin{array}{l}\text { Poor compared to } \\
\text { sym } 8 \text { and Db10 }\end{array}$ & $\begin{array}{l}\text { Poor compared to } \\
\text { Db4 and Sym }\end{array}$ & $\begin{array}{l}\text { Detects but not so } \\
\text { accurate compared } \\
\text { to } \mathrm{Db} 4\end{array}$ \\
\hline $\mathrm{Db}$ & $\begin{array}{l}\text { Db3 detects very } \\
\text { accurately }\end{array}$ & $\begin{array}{l}\text { Not accurately } \\
\text { compared to Dmey }\end{array}$ & $\begin{array}{l}\text { Poor compared to } \\
\text { Db10 and Sym } 8\end{array}$ & $\begin{array}{l}\text { Db4 detects sudden } \\
\text { sag but Gradual sags } \\
\text { are detected } \\
\text { accurately by Db10 } \\
\text { and Sym8 }\end{array}$ & $\begin{array}{ll}\mathrm{Db} 4 & \text { detects } \\
\text { accurately }\end{array}$ \\
\hline Sym8 & $\begin{array}{l}\text { Poor compared to } \\
\text { Db3 }\end{array}$ & $\begin{array}{l}\text { Not accurately } \\
\text { compared to Dmey }\end{array}$ & Detects accurately & Detects accurately & $\begin{array}{l}\text { Compared to } \mathrm{Db} 4 \text { it } \\
\text { is poor }\end{array}$ \\
\hline Coif & $\begin{array}{l}\text { Poor compared to } \\
\text { Db3 }\end{array}$ & $\begin{array}{l}\text { Not accurately } \\
\text { compared to Dmey }\end{array}$ & $\begin{array}{l}\text { Better but not } \\
\text { accurate like Sym } 8\end{array}$ & $\begin{array}{l}\text { Detects } \\
\text { accurately }\end{array}$ & $\begin{array}{l}\text { Poor compared to } \\
\text { Db4 and sym } 8\end{array}$ \\
\hline Bior & $\begin{array}{l}\text { Poor compared to } \\
\text { Db3 and other } \\
\text { wavelets }\end{array}$ & $\begin{array}{l}\text { Not accurately } \\
\text { compared to Dmey }\end{array}$ & $\begin{array}{l}\text { Not accurately } \\
\text { compared to Sym } 8\end{array}$ & $\begin{array}{l}\text { Not accurately } \\
\text { compared to Sym } 8\end{array}$ & $\begin{array}{l}\text { Not accurately } \\
\text { compared to } \mathrm{Db} 4\end{array}$ \\
\hline Haar & Poor & Poor & Poor & Poor & Poor \\
\hline
\end{tabular}

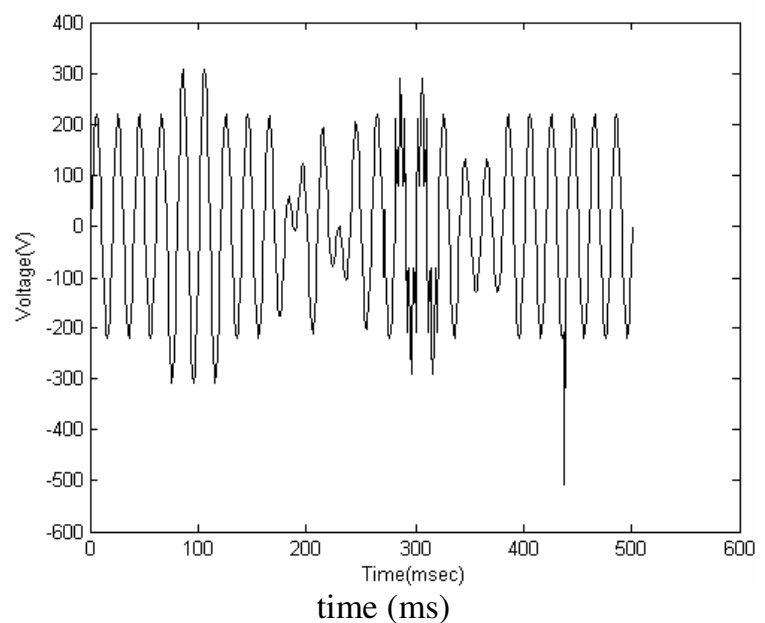

Fig. 3: Input signals containing the five disturbances simulated using MATLAB

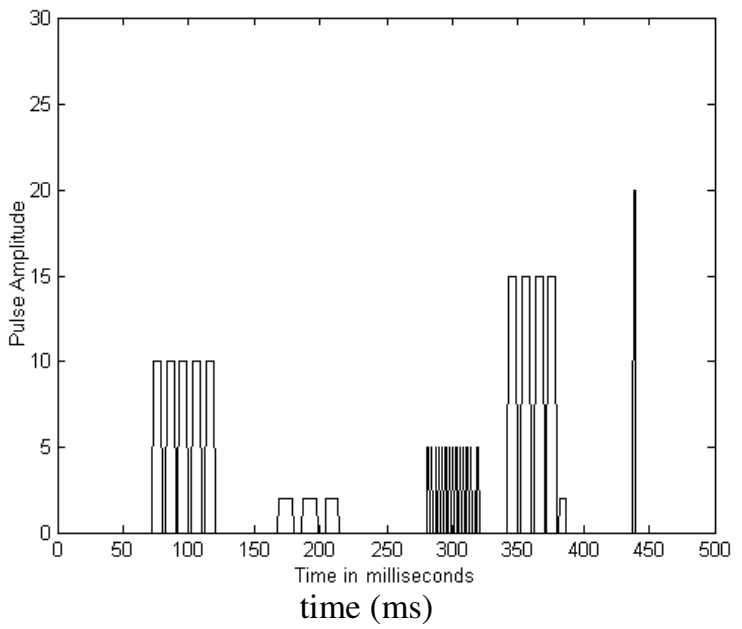

Fig. 4: Simulation results showing the presence of the power quality disturbances

In the proposed work, impulsive transient is considered for analysis. An impulsive transient is a sudden, non-power frequency change in unipolar voltage and current. The impulsive transients are mainly caused by lightning strikes. The waveform with impulsive transient occurring at $150^{\text {th }} \mathrm{ms}$ is shown in Fig. 2c.

The deviation from a perfect sine wave can be represented by harmonics, which are nothing but sinusoidal components having a frequency that is an integral multiple of the fundamental frequency. Harmonics, the by-products of power electronic converters are shown in Fig. 2d.

Voltage fluctuation, associated with visual "flicker" in lights, is a modulation of the fundamental component, caused by reactive load variations. The main causes of voltage flicker are the arc loads like arc furnace, arc welder and arc lamp. A typical fluctuating voltage is shown in Fig. 2e.

\section{RESULTS}

The proposed algorithm detects and classifies the power quality disturbances present in the input signal accurately. An input signal having all the five disturbances simulated in MATLAB considered as Case (i) is given as input to the detection algorithm. This is shown in Fig. 3 .The algorithm has accurately detected the disturbances and Figure 4 shows the accurate detection of power quality disturbances.

In the output of the detection algorithm for case (i),

Pulse amplitude $=10$ indicates the presence of voltage swell.

Pulse amplitude $=2$ indicates the presence of voltage flicker.

Pulse amplitude $=5$ indicates the presence of voltage harmonics.

Pulse amplitude $=15$ indicates the presence of voltage sag.

Pulse amplitude $=20$ indicates the presence of impulsive transient. 
Comparison evaluation of the detection capabilities of different wavelets: The following wavelets have been considered for comparison of performance evaluation using the proposed algorithm. They are DMeyer, Daubecius, Sym5, Coif, Bior and Haar wavelets as shown in Table I. From the table it is clear that, $\mathrm{Db} 3$ detects transients accurately.Db10 and sym8 detects voltage flicker accurately. The Dmey detects harmonics accurately and Db4 detects voltage Sag/Swell accurately.

\section{CONCLUSION}

Power system events may be classified by quantity and duration of power quality disturbances. This paper has presented a novel method to detect and classify disturbed voltage which works for any number of cycles and can be customized for any sampling rate. This novel detection algorithm shows promise for the future development of fully automated monitoring systems with classification ability. A detailed comparative evaluation of the performance capabilities of different wavelets using the proposed algorithm is also done.

\section{REFERENCES}

1. Malay, S. and W.L. Hwang, 1992. Singularity detection and processing with wavelets. IEEE Trans. Information Theory, 38: 617-643.
2. Ghosh, A. and G. Ledwich, 2002. Power Quality Enhancement Using Custom Power Devices. Kluwer Academic Publishers, USA.

3. Santoso, S., E.J. Powers, W.M. Grady and A.C. Parsons, 2000. Power quality disturbance waveform recognition using wavelet-based neural classifier. I. Theoretical foundation. IEEE Trans. Power delivery, 15: 222-228.

4. Wilkinson, W.A. and M.D. Cox, 1996. Discrete wavelet analysis of power system transients. IEEE Trans. Power Systems, 11: 2033-2044.

5. Heydt,G.T. and A.W. Galli, 1997. Transient power quality problems analyzed using wavelets. IEEE Trans. Power Delivery, 12: 908-915.

6. Angirisani, L., P. Daponte, M. D'Apuzzo and A. Testa, 1998. A measurement method based on the wavelet transform for power quality analysis. IEEE Trans. on Power Delivery, 13: 990-998.

7. Perunicic, B., M. Mallini, Z. Wang and Y. Liu, 1998. Power quality disturbance detection and classification using wavelets and artificial neural networks. Proc. Harmonics and Quality of Power, 1: 77-82. 